\title{
THE IMPACT OF NEW WATER VAPOR SPECTROSCOPY ON SATELLITE RETRIEVALS
}

\author{
A. N. MAURELLIS \\ SRON National Institute for Space Research, Sorbonnelaan 2, \\ 3584 CA Utrecht, The Netherlands \\ R. LANG, J. E. WILLIAMS and W. J. VAN DER ZANDE \\ FOM-Institute for Atomic and Molecular Physics, Kruislaan \\ 407, 1098 SJ Amsterdam, Amsterdam, The Netherlands \\ K. SMITH and D. A. NEWNHAM \\ CCLRC Rutherford Appleton Laboratory, Oxon, OX11 0QX, \\ The United Kingdom \\ J. TENNYSON and R. N. TOLCHENOV \\ Department of Physics and Astronomy, University College Lon- \\ don, Gower Street, London WC1E 6BT, The United Kingdom
}

\begin{abstract}
Water vapor, arguably the most important trace gas constituent of Earth atmospheric physics, is also both a retrieval goal and a hindrance in the retrievals of other trace gases from nadir-measuring satellite spectrometers. This is because the atmospherically-attenuated solar spectrum in the visible and shortwave infrared is littered with water vapor bands. The recent plethora of water vapor spectroscopy databases in this spectral region has prompted us to study their utility in satellite retrievals. We consider water vapor spectroscopy compiled from four sources including new spectroscopy due to University College London and Imperial College London. Radiative transfer models of satellite measurements, in combination with accurate retrieval techniques, are quite sensitive to the accuracy and completeness of the water vapor spectroscopy. Notwithstanding the high degree of variability of a number of different factors in satellite measurements we show that retrievals are sensitive to database differences which suggests that our knowledge of water vapor spectroscopy is not as yet complete. In addition, new laboratory measurements indicate that the role of both the far-line wings of water vapor and the cumulative effect of many weak lines each have an important role to play in forming the so-called continuum.
\end{abstract}




\section{Introduction}

Oddly enough, a quantification of broadband absorption effects in atmospheric radiative transfer modeling is closely linked to an accurate knowledge of line spectra, especially those of water vapor which absorbs significantly in the atmosphere at most wavelengths longward of about 400 nm.

Many instances of water vapor line signatures may be found in visible and shortwave infrared spectra taken by nadir-sounding instruments such as the European Space Agency's Global Ozone Monitoring Experiment (GOME) on board the ERS-2 platform (Noël et al., 2002) and the Scanning Imaging Absorption Spectrometer for Atmospheric Chartography (SCIAMACHY) on board ESA's recently-launched ENVISAT platform (Bovensmann et al., 1999). In this work we present retrieval simulations for these instruments using rovibrational overtone bands of water vapor in the visible and shortwave infrared and spectroscopy from four different sources:

- new data (hereafter UCL-IC) due to a collaboration between University College London and Imperial College, and based on new laboratory measurements at the Molecular Spectroscopy Facility, Rutherford Appleton Laboratory (RAL) (Schermaul et al., 2002);

- new data (hereafter ULB-UFR) due to a collaboration between Laboratoire de Chimie Physique Moléculaire at the Université Libre de Bruxelles, the Institut d'Aéronomie Spatiale de Belgique, and the Groupe de Spectrométrie Moléculaire et Atmosphérique, UFR Sciences, Reims (Carleer et al., 1999; Coheur et al., 2002).

- the HITRAN-1996 (Rothman et al., 1998) and HITRAN-2000 (Giver et al., 2000) databases;

- the ESA-WV- (B) and (R) databases (Learner et al., 2000; Schermaul et al., 2001);

We show here that synthetic spectra generated from these line parameter databases and a knowledge of the background atmosphere may be used in combination with satellite-measured spectra to fix requirements for line intensity accuracies in spectral databases. Thus it becomes possible to use the atmosphere as a long pathlength laboratory in order to place limits on the levels of spectroscopic accuracy required for water vapor retrieval. This has additional, important consequences for our understanding of the water vapor continuum which are supported by other modeling efforts as well as by recent laboratory measurements of the water vapor continuum at RAL. 


\section{The Atmosphere as Laboratory}

In order to compare modeled and satellite-measured earth-backscattered solar radiances we define a correction to the ratio between modeled and measured reflectivity spectra in terms of uncertainties in cross-section line intensities, averaged over the spectral region covered by each detector pixel in the focal plane of a grating spectrometer array. First we assume that the relative error in the cross-section corresponding to the spectral region covered by the satellite detector pixel, $\hat{\sigma}$, is dominated by the relative error in the pixel-averaged line intensity so that

$$
\frac{\Delta \hat{\sigma}}{\hat{\sigma}}=\frac{\Delta S}{S} \equiv-\Delta \phi .
$$

The quantity $\Delta \phi$ represents the correction required to bring the crosssection using the line intensity values of the spectral database into agreement with the satellite-measured average cross section. Thus (Lang et al., 2002)

$$
R_{\mathrm{sat}}=R_{\bmod } \exp \left(-\frac{\Delta S}{S} \int_{s} \hat{\sigma}_{\bmod }(s) n(s) d s\right),
$$

where $R_{\text {sat }}$ is the measured reflectivity. The modeled reflectivity and crosssection are given by $R_{\text {mod }}$ and $\sigma_{\text {mod }}$, respectively.

Figures 1 and 2 show how this quantity may be used to distinguish directly between spectral databases. There is a caveat; this approach does not exclude the possibility that detector pixel electronics offsets could also contribute a residual signature which is comparable to that of the spectral database uncertainties. There is no way to exclude this possibility in the case of GOME. However SCIAMACHY has the capability to make so-called "dark" measurements which can be used to "flatfield" each detector pixel to high precision.

\section{Retrieval Theory}

Standard retrieval theory (Eriksson, 2000), begins with a forward model $\mathcal{T}$ which dictates the passage of radiation through the atmosphere and instrument, viz.

$$
y=\mathcal{T}(x ; b, S)+\varepsilon,
$$

where $y$ is the measured spectrum, $x$ reflects the true state of the atmosphere, $\varepsilon$ is the shotnoise on the measurement, and the two other arguments of the forward model constitute a set of instrumental parameters $b$ and spectroscopic parameters $S$. Retrieval is the process of formally inverting 

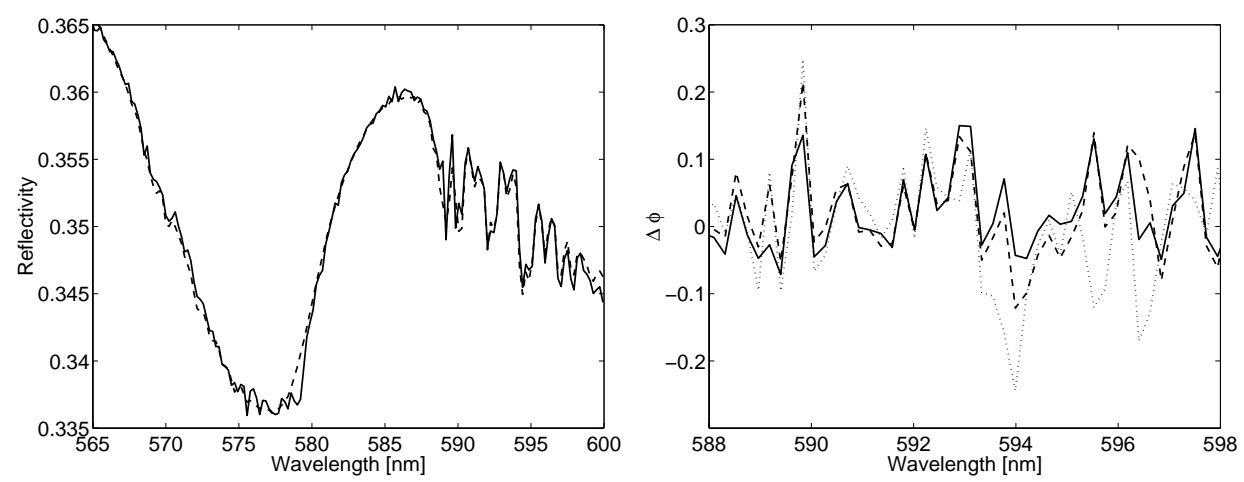

Figure 1. Left Panel Comparison of a GOME-measured reflectivity (solid curve) with the results of a line-by-line calculation (dashed curve). The background consists of the $\left(\mathrm{O}_{2}\right)_{2}$ collision-induced absorption complex (broad feature centered at $577 \mathrm{~nm}$ ), part of the $\mathrm{O}_{3}$ Chappuis band throughout the spectral window and a sharp feature at about $590 \mathrm{~nm}$ due to the sodium lines. Right Panel Pixel-to-pixel variation of the residual mismatch $\Delta \phi$ calculated for three different GOME measurements using a line-by-line forward model (Lang et al., 2002) corresponding to a high (solid line), medium (dashed line) and low (dotted line) water vapor column. The measurements were taken at significantly different geolocations and very different solar zenith angles $\left(37^{\circ}, 23^{\circ}, 73^{\circ}\right.$, respectively). Notwithstanding differences in surface albedo, aerosol content and the contribution of multiple scattering to the measurements the three curves show similar patterns which could imply either detector biases or spectroscopic biases are present in the modeling.

Eqn. (3) in order to determine a set of trace gas profiles, notated by $\hat{x}$, which satisfy a modeled spectrum

$$
\hat{y}=\mathcal{T}(\hat{x} ; b, S) .
$$

The problem is generally ill-posed, since the number of elements in the measurement vector is usually much larger than the number of retrievable profile points (see DFS below). Thus even though $|\hat{y}-y| \leq \varepsilon$ may result from a good inversion, $\hat{x}$ will almost never be identical to $x$ simply because there may be insufficient information in the measurement or because information has been lost due to the spatial and spectral discretization of the model as well as any spectral smoothing caused by the spectrometer (so-called nullspace errors). In addition, there may be uncertainties in the radiation transport model and errors in instrumental parameters $b$ which, at least in the latter case, are predictable through careful calibration of the instrument. Both of the last two errors are avoided in what follows by carrying out retrievals from simulated spectra which are generated using the same atmospheric and instrument physics as the retrieval. This allows us to focus primarily in this work on the error in the forward model due to spectroscopic uncertainties, in particular, the effects of uncertainty $(\delta S)$ 

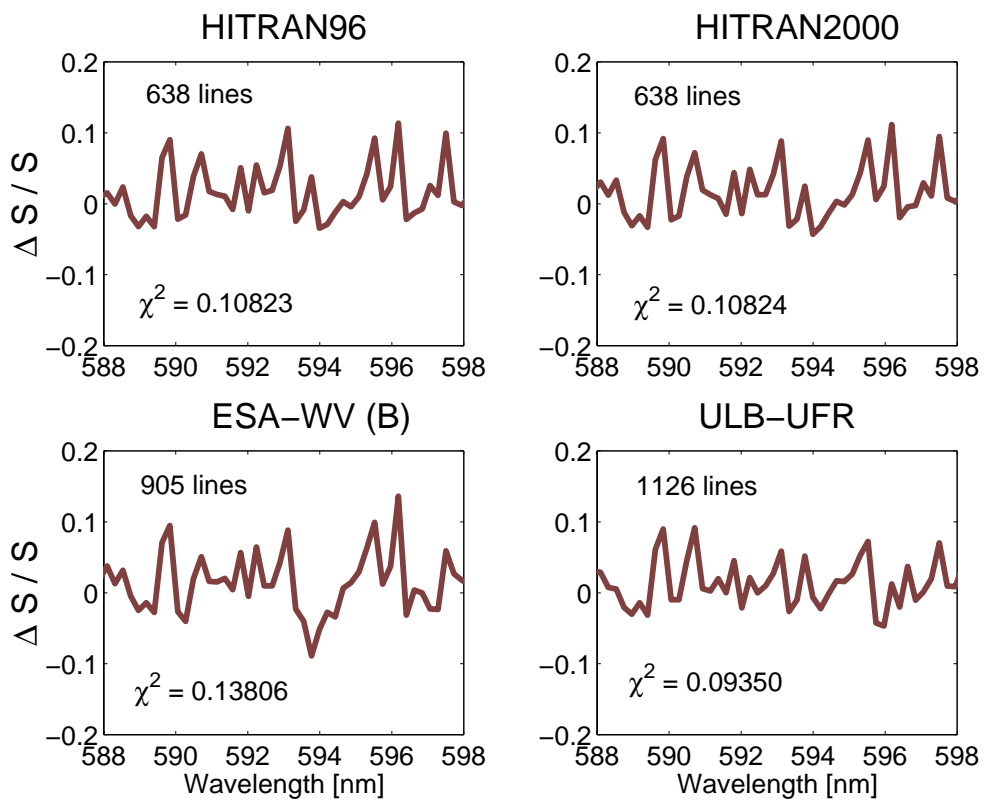

Figure 2. Determination of cross-section correction for different spectral databases in the region of the $5 \nu$ polyad using real GOME data, as per Figure 1 and Eqn. (2). This confirms the sensitivity of the retrieval to small spectroscopic differences on the order of $10 \%$. Each plot shows the correction derived from a model fit to the data which uses a different source of line parameters: HITRAN-1996; HITRAN-2000; ESA-WV (B only); ULB-UFR. The number of water vapor lines contained in each database in this spectral window is also shown. At least for the $5 \nu$ polyad HITRAN data appears to fare reasonably well, though the ULB data comes out better. The ESA-WV(B) data fares poorly, as expected, since this portion of the ESA-WV database mixes theoretical values and data derived from other studies independent of the ESA-WV study. In addition, it should be noted that the databases are not entirely independent of one another. ULB-UFR lines have intensities from (Carleer et al., 1999; Coheur et al., 2002) but are assigned from (Partridge and Schwenke, 1997; Polyansky et al., 1998; Zobov et al., 2000) and ESA-WV (R). ESA-WV(B) line assignments are made using (Zobov et al., 2000) but intensities are taken from HITRAN 2000 with some assignments from (Carleer et al., 1999).

in $S$ (mean line intensity per wavelength grid cell as in Eqn. (1)). The net contribution to the total retrieval errors may then be written as

$$
\delta x=[A-I](\hat{x}-x)+\frac{\partial \hat{x}}{\partial y} \varepsilon+\frac{\partial \hat{x}}{\partial y} \frac{\partial \mathcal{T}}{\partial S} \delta S .
$$

Here the first term encompasses the contribution to retrieval uncertainty of the nullspace errors, the second the shotnoise error and the third the mean line intensity parameter error. The first term includes the so-called 

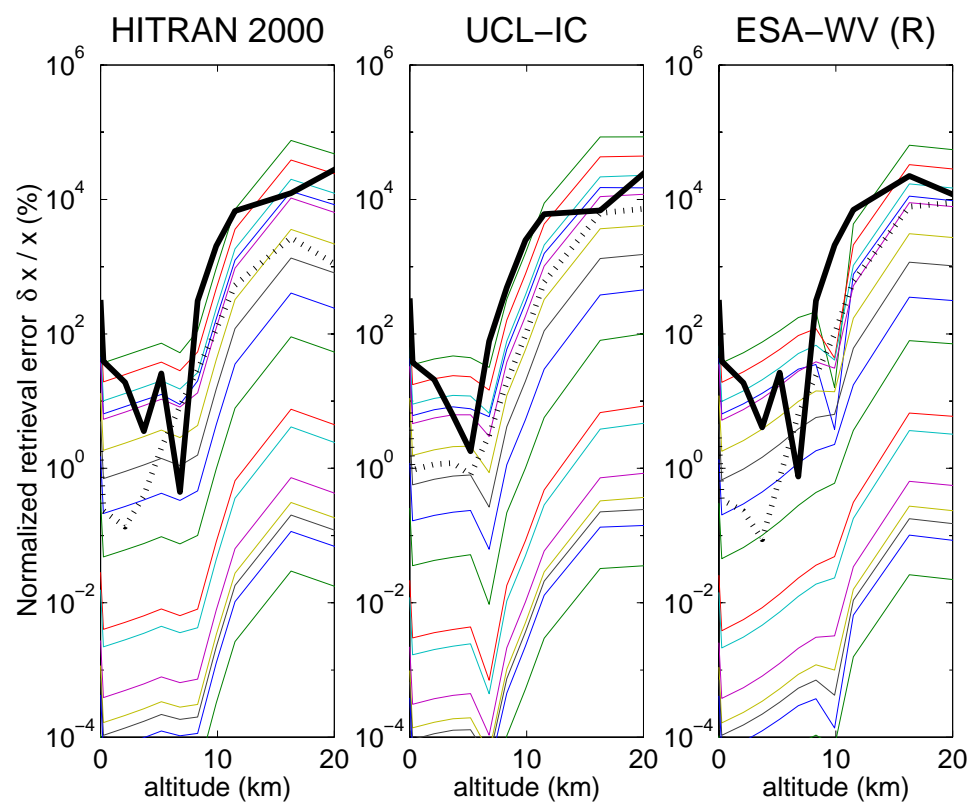

Figure 3. Profile retrieval errors (cf. Eqn. (5)) derived from simulations assuming a single nadir-gazing measurement and using the spectroscopy in three of the databases for the $3 \nu+\delta$ polyad. Although a single nadir measurement in a single absorption band would seldom be deemed sufficient for a good profile retrieval it is nonetheless useful to simulate the resulting errors as a function of altitude. The solid line indicates the effect of nullspace errors, the dotted line the effect of the detector shotnoise error and the many colored lines the effect of line intensity uncertainties on the order of $10 \%$ for each of an array of simulated detector pixels. The effects of spectroscopic uncertainties are still more significant than other sources of error, especially for altitudes below 15 $\mathrm{km}$, where the bulk of the water vapor column is situated. The much higher relative uncertainties at altitudes above $15 \mathrm{~km}$ derive from the much lower water vapor densities at these altitudes.

averaging kernel of the inversion

$$
A=\frac{\partial \hat{x}}{\partial y} \frac{\partial \mathcal{T}}{\partial x} .
$$

A wavelength grid cell is assumed, for the purposes of this discussion, to be the wavelength region covered by a detector pixel. Matrix $A$ can also be used to calculate the degrees of freedom for signal in the retrieval (abbreviated to DFS), via

$$
\operatorname{DFS}=\operatorname{trace}(A) .
$$

The profile weighting function (or dependence of the forward model on the profile) $\partial \mathcal{T} / \partial x$ may be calculated trivially as a function of altitude if one assumes a simple Beer's law for atmospheric extinction of light. The 
spectroscopic parameter weighting function $\partial \mathcal{T} / \partial S$ is harder to calculate in general. However, if one assumes that the total spectral structure sampled by a detector pixel may be represented by a giant-line approximation such as the Spectral Structure Parameterization (Maurellis et al., 2000) then one may express the mean transmittance corresponding to each detector pixel as

$$
\mathcal{T}=1+w \exp (-S N)-w
$$

where $S$ is the line intensity of the giant line for a layer of absorbing gas with column $N$ and $w$ is the line width. The spectroscopic parameter weighting functions follow immediately on partial differentiation with respect to $S$ or $w$. Finally, the mean transmittance must be further smoothed in order to simulate the spectrometer slit and so to obtain the modeled reflectivity, $\hat{y}$.

\section{Results of Simulations}

Figure 3 summarizes some nadir profile retrieval simulation results for the different databases. The implicit assumption is that each database is complete for the simulated atmosphere. The black curves show the effect on the retrieved profile, in percentage of the true profile and as a function of altitude, of the nullspace and shotnoise uncertainties induced by the errors included in Eqn. (5). The colored lines correspond to the effect of a $10 \%$ average line intensity $S$ uncertainty for each detector pixel wavelength grid cell in the $3 \nu+\delta$ polyad $\left(11,600-12,750 \mathrm{~cm}^{-1}\right)$ (i.e. $\delta S=0.1 S$, cf. Eqn. (1) and the maximum uncertainties in Figure 2 ). The forward model assumes simple Beer's law extinction, a surface albedo of $30 \%$, a solar zenith angle of $30^{\circ}$ and a mid-latitude summer water vapor profile, with total column of $1.24 \times 10^{23}$ molecules $\mathrm{cm}^{-2}$. The simulated spectra are smoothed with a Gaussian function with FWHM corresponding to a slit of $0.2 \mathrm{~nm}$, typical for satellite grating spectrometers in this wavenumber range. Clearly the effects of spectroscopic uncertainty (colored lines) are frequently comparable to and occasionally exceed the other sources of error, especially at the important lower altitudes which correspond to the bulk of the water vapor column.

An increase in the requirement on spectral uncertainty from $10 \%$ to $1 \%$ would place all the colored curves a factor of 10 lower, with the result that the retrieval would be optimal, i.e. nullspace- and shotnoise-limited. There is also a change in the shape of the averaging kernels (see Figure 4) as lines are removed from the retrieval (assuming a full set of lines in each simulated measurement). The sensitivity of the retrieved water vapor profile to the true profile at higher altitudes drops dramatically when lines with intensity levels less than $5 \times 10^{-25} \mathrm{~cm} /$ molecule are excluded from the retrieval (but not from the forward model used to calculate the simulated 

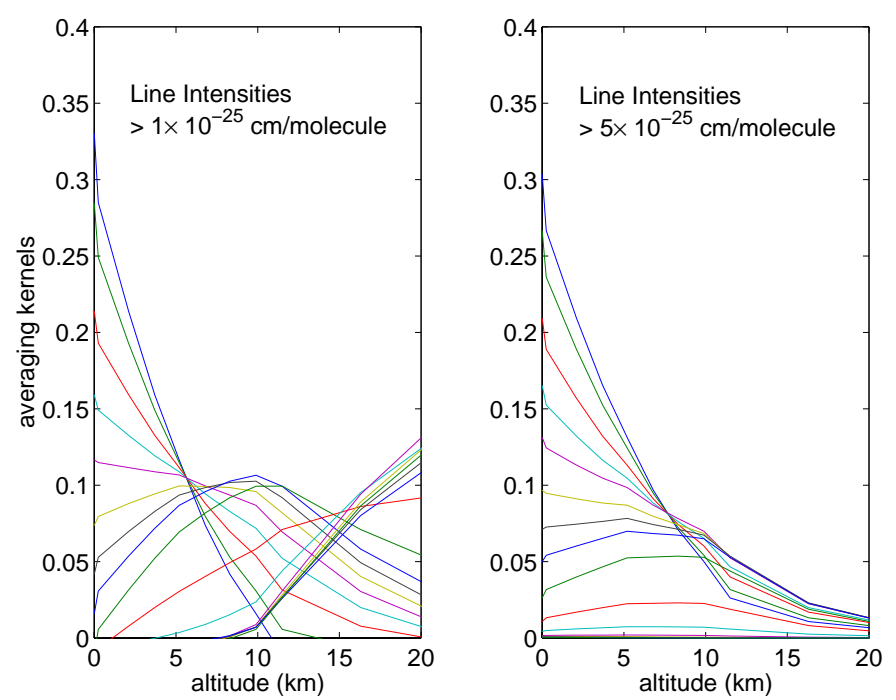

Figure 4. Two sets of averaging kernels (row vectors of the matrix $A$ ) for a set of simulations using the ESA-WV database. (The averaging kernels give an indication of the sensitivity of the retrieved profile to the true profile.) Successive levels of weak line intensity were removed from the ESA-WV database starting with all the lines in the database until a significant change in the averaging kernels resulted (a drop in the retrieval sensitivity above $7 \mathrm{~km}$ ). This change dictates a level in line intensity above which line intensities have to be known to high accuracy and below which the completeness of the spectral database will contribute to a continuum signal but for which a high accuracy of line intensity parameters is probably not crucial.

measurement). Together these indicate that line intensities greater than about $5 \times 10^{-25} \mathrm{~cm} /$ molecule should be known to an accuracy on the order of $1 \%$ or better and the completeness of the databases below this level should be well established since this will contribute to the overall level of the assumed broadband or continuum signal. We note in passing that this level corresponds roughly to the range, $1-6 \times 10^{-24} \mathrm{~cm} /$ molecule, below which line intensity differences between UCL-IC and HITRAN-2000 become significant (Schermaul et al., 2002)). In addition the increase in total number of weak lines in the new databases yields an increase in DFS value from approximately 3 (when using the HITRAN2000 database) to 5-7 (for the ESA-WV and UCL-IC databases) which implies that the new databases have the potential to substantially enhance retrievals. Figure 5 shows the results of doing column retrievals from real GOME data using two of the databases, HITRAN-1996 and UCL-IC. Notwithstanding difficult remote sensing issues to be dealt with in this wavelength region (not least of which is a considerable change in the surface albedo of vegetation at approximately $720 \mathrm{~nm}$ ) the effects on column retrieval of using different 


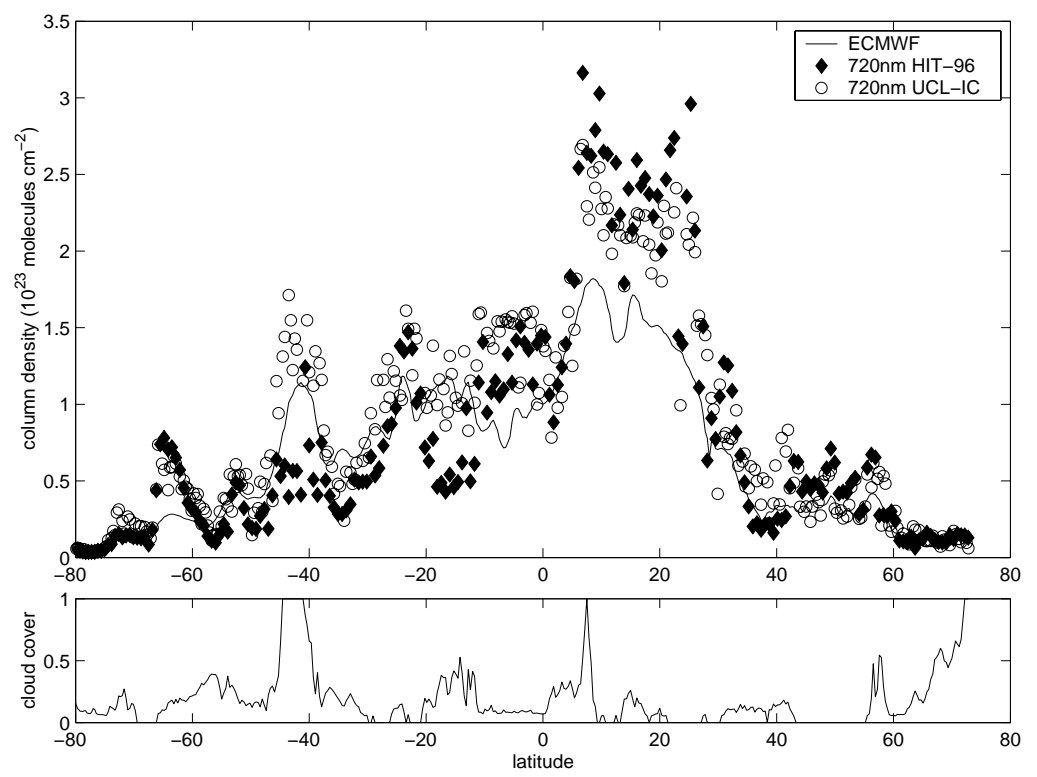

Figure 5. Water vapor column retrievals (upper panel) using the HITRAN-1996 (filled diamonds) and UCL-IC (circles) databases for the $4 \nu$ polyad (centered at approximately $720 \mathrm{~nm}$ ). The columns have been retrieved from measurements across a single north-south track of GOME data (see horizontal axis). The solid line shows water vapor columns taken from the European Center for Medium-Range Weather Forecasting (ECMWF) and is provided purely for comparison purposes, as is the measure of cloud cover (lower panel) (from 0 for no cloud cover to 1 for fully-clouded) inferred from other GOME data.

spectral databases are quite significant. However it is still too early to decide whether or not using the new database constitutes an improvement.

\section{New Laboratory Measurements of the Water Vapor Continuum}

The distinction between broadband effects due to large numbers of low intensity lines on the one hand and true continuum effects on the other has been alluded to, above, in terms of completeness of the spectral line list used in a given radiative transfer model. Recently this distinction was made in the laboratory using a combination of a high-resolution Fourier transform spectrometer and two variable path-length absorption cells at RAL. Comparisons between modeled and measured laboratory transmittance spectra at two temperatures $(296$ and $342 \mathrm{~K})$ in the spectral region $4400-6000 \mathrm{~cm}^{-1}(1.6-2.3 \mu \mathrm{m})$ indicate that absorption by pure water vapor is underestimated between $4900 \mathrm{~cm}^{-1}$ and $5700 \mathrm{~cm}^{-1}$. Figure 6 shows results from the short path-length absorption cell (SPAC) operated at an optical path length of $9.74 \mathrm{~m}$ and a temperature of $342.1 \mathrm{~K}$. The measure- 
ments were modeled using the Reference Forward Model line-by-line code (Dudhia, 1997) in conjunction with one or the other of the CKD version 2.4 continuum (cf. Clough, this volume and (Clough et al., 1989)) and the continuum cross sections from new far-line wing calculations for water vapor (Ma \& Tipping, 2002). Observed and calculated absorbances were compared by subdividing each absorption band into $5 \mathrm{~cm}^{-1}$ wide intervals. In each interval a baseline offset was determined by a non-linear least squares fit of the modeled spectrum to the measurement. Only those data where the calculated and observed Napierian absorbances were below 1.0 were used in the comparisons to avoid introducing spectral saturation errors. As shown in Figure 6 neither of the two water vapor continuum treatments fully accounts for the observed additional absorption. The residual absorption is ostensibly reduced the most by the use of the CKD continuum in the forward model. Future investigations at RAL will center on the role of metastable water vapor dimers as a possible cause for the discrepancy between observations and calculations. However, since the CKD continuum is an empirically-adjusted broadband offset rather than a continuum calculation derived from first principles, one likely conclusion is that CKD accounts for the net contribution of many missing water vapor lines, the cumulative effect of which swamps any true continuum effect.

\section{Conclusions}

Thus it turns out that the addition of weak lines to the current databases is probably crucial for explaining inconsistences between laboratory measurements and models as well as improving the accuracy of retrievals in general. The role of weak lines in contributing, effectively, to a continuum was further corroborated recently by a study of the radiative forcing effect due to different water vapor spectral databases (Zhong et al., 2001). This study concluded that large numbers of weak water vapor lines such as those included in the ESA-WV database (but not in the HITRAN databases) could account for as much as $90 \%$ of the CKD continuum.

Retrievals, both real and simulated, have shown that sufficient levels of cross-section accuracy have not yet been attained. The indication is that strong lines should be known to accuracies of $1 \%$ or better. This will hopefully be met by future improvements to the UCL-IC database due to the availability of new, more accurate potential surfaces and dipole moment calculations. The temperature dependence of air-broadening coefficients, although not discussed here, is also important and will be addressed in a future study.

Thus it is possible to verify spectroscopic databases using high-resolution laboratory as well as medium-resolution satellite measurements. Another 

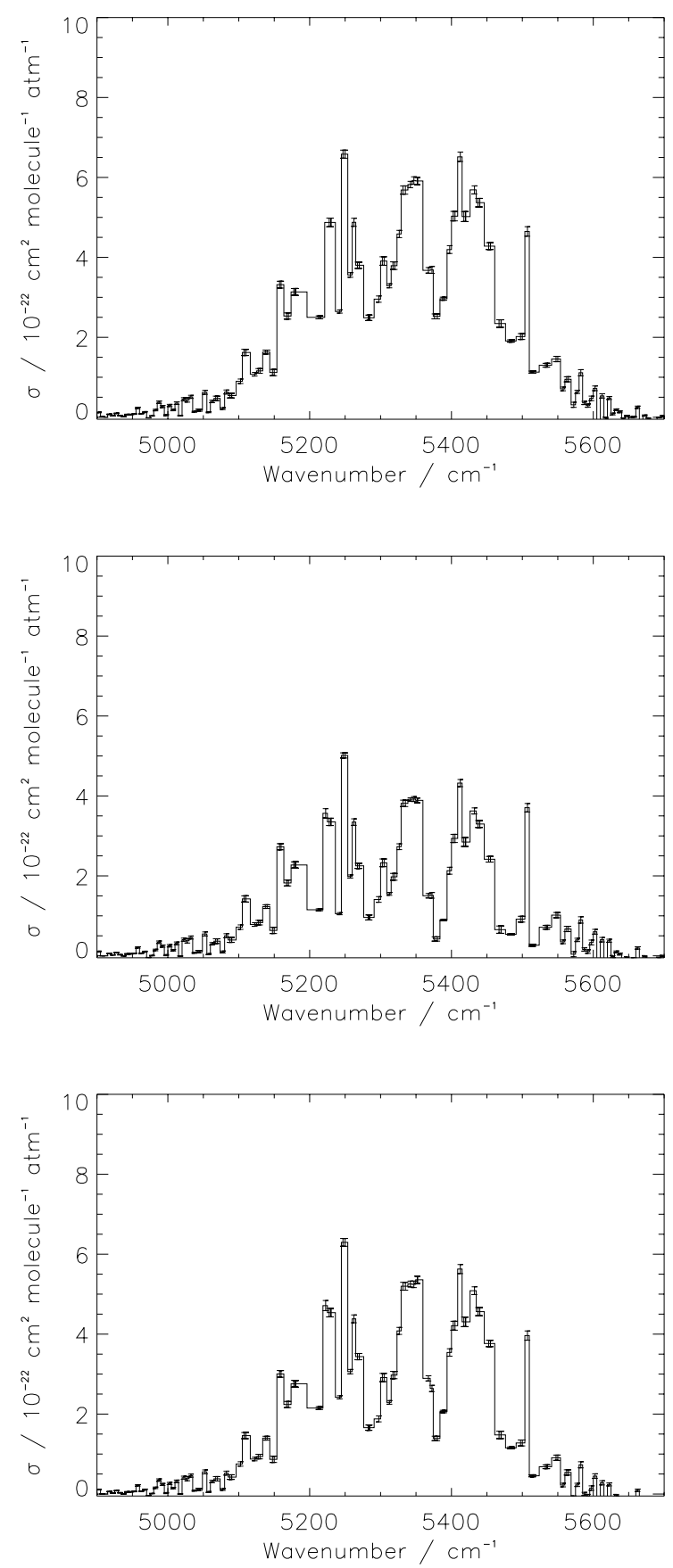

Figure 6. Residuals in absorption cross-sections between RAL SPAC measurements and spectra generated by the RFM/HITRAN line-by-line model in $5 \mathrm{~cm}^{-1}$ intervals between $4900 \mathrm{~cm}^{-1}$ and $5700 \mathrm{~cm}^{-1}$ for $100 \mathrm{hPa}$ of pure water vapor at $342 \mathrm{~K}$. Top panel Results from a pure line-by-line calculation. Error bars indicate all known experimental uncertainties. Center panel Results from a RFM/HITRAN calculation assuming a Voigt line shape up to $25 \mathrm{~cm}^{-1}$ on either side of the line centre and the CKD 2.4 water vapor continuum. Lower panel As in the other panels except that the RFM/HITRAN calculation assumed a Voigt line shape up to $10 \mathrm{~cm}^{-1}$ on either side of the line centre and the far-line wing continuum due to Tipping and Ma beyond $10 \mathrm{~cm}^{-1}$. 
way is to use high-resolution ground-based measurements of atmospheric absorption (Smith et al., 2001; Veihelmann et al., 2002). In any case, we have at our disposal a unique long pathlength laboratory setup-the atmosphere-providing we can use it correctly!

\section{Acknowledgements}

We thank Ilse Aben (SRON) for being among the first to recognize the importance of spectral residual analysis based on satellite measurements. ESA is acknowledged for providing GOME data processed by DFD/DLR. NERC and EPSRC are also acknowledged for additional support.

\section{References}

Bovensmann H., J. P. Burrows, M. Buchwitz, J. Frerick, S. Noel, V. V. Rozanov, K. V. Chance, A. P. H. Goede. SCIAMACHY: Mission objectives and measurement modes. Journal of the Atmospheric Sciences, 56(2):127-150, 1999.

Carleer M., A. Jenouvrier, A. C. Vandaele, P. F. Bernath, M. F. Merienne, R. Colin, N. F. Zobov, O. L. Polyansky, J. Tennyson and V. A. Savin. The near infrared, visible, and near ultraviolet overtone spectrum of water. Journal of Chemical Physics, 111(6):24442450, 1999.

Clough, S. A., F. X. Kneizys and R. W. Davies. Line shape and the water vapor continuum. Atmospheric Research, 23:229-241, 1989.

Coheur P. F., S. Fally, M. Carleer, C. Clerbaux, R. Colin, A. Jenouvrier, M. F. Merienne, C. Hermans and A.C. Vandaele. New water vapor line parameters in the 26 000-13 000 $\mathrm{cm}^{-1}$ region. Journal of Quaintitative Spectroscopy \& Radiative Transfer, 74(4):493$510,2002$.

A. Dudhia. Reference forward model version 3: Software user's manual. European Space Technology Centre (ESTEC) Document PO-MA-OXF-GS-0003, European Space Agency, Paris, France, 1997.

Eriksson, P. Analysis and comparison of two linear regularization methods for passive atmospheric observations. Journal of Geophysical Research, 105(D14):18,157-18,167, 2000 .

Giver, L. P., C. Chackerian and P. Varanasi. Visible and near-infrared $\left(\mathrm{H}_{2}^{16} \mathrm{O}\right.$ line intensity corrections for HITRAN-96. Journal of Quaintitative Spectroscopy 83 Radiative Transfer, 66(1):101-105, 2000.

Lang, R., A. N. Maurellis, W. J. van der Zande, I. Aben., J. Landgraf and W. Ubachs. Forward Modelling and Retrieval of Water Vapor from the Global Ozone Monitoring Experiment: Treatment of Narrowband Absorption Spectra. Journal of Geophysical Research, 107(D16), 4300, doi:10.1029/2001JD001453, 2002.

Learner, R. C. M., R. Schermaul, J. Tennyson, N. F. Zobov, J. Ballard, D. Newnham and M. G. Wickett. Measurement of Water Absorption Cross-Sections for the Exploitation of GOME data. ESTEC Contract No. 13312/9/NL/SF.

$\mathrm{Ma}, \mathrm{Q}$. and R. Tipping. The frequency detuning correction and the asymmetry of line shapes: The far wings of $\mathrm{H}_{2} \mathrm{O}-\mathrm{H}_{2} \mathrm{O}$. Journal of Chemical Physics, 116(1):4102-4115.

Maurellis, A. N., R. Lang and W. J. van der Zande. A new DOAS parameterization for retrieval of trace gases with highly-structured absorption spectra. Geophysical Research Letters, 27(24):4069-4072, 2000. 
Noël S., M. Buchwitz, H. Bovensmann and J. P. Burrows. Retrieval of total water vapor column amounts from GOME/ERS-2 data. Advances in Space Research, 29(11): 1697-1702, 2002.

Partridge, H. and D. W. Schwenke. The determination of an accurate isotope dependent potential energy surface for water from extensive ab initio calculations and experimental data. Journal of Chemical Physics, 106(11):4618-4639, 1997.

Polyansky O. L., N. F. Zobov, S. Viti and J. Tennyson. Water vapor line assignments in the near infrared. Journal of Molecular Spectroscopy, 189(2):291-300, 1998.

Rothman, L. S. and 19 authors The HITRAN molecular spectroscopic database and HAWKS (HITRAN Atmospheric Workstation): 1996 edition. Journal Quaintitative Spectroscopy $\& 3$ Radiative Transfer, 60(5):665-710, 1998.

Schermaul R., R. C. M. Learner, D. A. Newnham, J. Ballard, N. F. Zobov, D. Belmiloud and J. Tennyson. The water vapor spectrum in the region $8600-15000 \mathrm{~cm}^{-1}$ : Experimental and theoretical studies for a new spectral line database. I. Linelist Construction Journal of Molecular Spectroscopy, 208(1):43-50, 2001.

Schermaul R., R. C. M. Learner, A. A. D. Canas, J. W. Brault, O. L. Polyansky, D. Belmiloud, N. F. Zobov, J. Tennyson. Weak line water vapor spectra in the region 13 200-15 $000 \mathrm{~cm}^{-1}$. Journal of Molecular Spectroscopy, 211(2):169-178, 2002.

Smith, K. M. and D. A. Newnham, High-resolution atmospheric absorption by water vapor in the 830-985 $\mathrm{nm}$ region: Evaluation of spectroscopic databases. Geophysical Research Letters, 28(16):3115-3118, 2001.

Veihelmann, B., R. Lang, K. M. Smith, D. A. Newnham and W. J. van der Zande Evaluation of spectroscopic databases of water vapor between 585 and $600 \mathrm{~nm}$. Geophysical Research Letters, 29(15), 1752, doi:10.1029/2002GL015330, 2002.

Zobov, N. F., D. Belmiloud, O. L. Polyansky, J. Tennyson, S. V. Shirin, M. Carleer, A. Jenouvrier, A.-C. Vandaele, P. F. Bernath, M. F. Mérienne and R. Colin. The near ultraviolet rotation-vibration spectrum of water. Journal of Chemical Physics, 113(4):1546-1552, 2000.

Zhong, W., J. D. Haigh, D. Belmiloud, R. Schermaul and J. Tennyson. The impact of new water vapor spectral line parameters on the calculation of atmospheric absorption. Quarterly Journal of the Royal Meteorological Society, 127:1615-1626, 2001. 
maurellis_02_v3.tex; 29/10/2002; 15:40; p.14 